\title{
Moutard transform for the generalized analytic functions *
}

\author{
P.G. Grinevich ${ }^{\dagger} \quad$ R.G. Novikov ${ }^{\ddagger}$
}

\begin{abstract}
We construct a Moutard-type transform for the generalized analytic functions. The first theorems and the first explicit examples in this connection are given.
\end{abstract}

\section{Introduction}

We consider the equation

$$
\partial_{\bar{z}} \psi=u \bar{\psi} \text { in } D \subseteq \mathbb{C},
$$

where $u=u(z)$ is a given function in $D, D$ is open in $\mathbb{C}$. The functions $\psi=\psi(z)$ satisfying (11) are known as generalized analytic functions in $D$. In

${ }^{*}$ The main part of the work was fulfilled during the visit of the first author to the Centre de Mathématiques Appliquées of École Polytechnique in October 2015. The first author was partially supported by the Russian Foundation for Basic Research, grant 1301-12469 ofi-m2, by the program "Leading scientific schools" (grant NSh-4833.2014.1), by the program "Fundamental problems of nonlinear dynamics".

${ }^{\dagger}$ L.D. Landau Institute for Theoretical Physics, pr. Akademika Semenova 1a, Chernogolovka, 142432, Russia; Lomonosov Moscow State University, Faculty of Mechanics and Mathematics, Russia, 119991, Moscow, GSP-1, Leninskiye Gory 1, Main Building; Moscow Institute of Physics and Technology, 9 Institutskiy per., Dolgoprudny, Moscow Region, 141700, Russia; e-mail: pgg@landau.ac.ru

${ }^{\ddagger}$ CNRS (UMR 7641), Centre de Mathématiques Appliquées, École Polytechnique, 91128, Palaiseau, France; IEPT RAS, 117997, Moscow, Russia; e-mail: novikov@cmap.polytechnique.fr 
this article the notation $f=f(z)$ does not mean that $f$ is holomorphic. In the literature it is usually assumed that

$$
\begin{array}{lll}
u \in L_{p}(D), & p>2, & \text { if } D \text { is bounded, } \\
u \in L_{p, 2}(\mathbb{C}), & p>2, & \text { if } D=\mathbb{C},
\end{array}
$$

where

$$
\begin{aligned}
& L_{p, \nu}(\mathbb{C}) \text { denotes complex-valued functions } u \text { such that } \\
& u \in L_{p}\left(D_{1}\right), \quad u_{\nu} \in L_{p}\left(D_{1}\right), \text { where } u_{\nu}(z)=\frac{1}{|z|^{\nu}} u\left(\frac{1}{z}\right), \\
& D_{1}=\{z \in \mathbb{C}:|z| \leq 1\} .
\end{aligned}
$$

The theory of generalized analytic functions is presented in [2], [12]. However, to the best of our knowledge, algebraic Moutrad-type transforms, going back to [6] were not yet considered in the framework of this theory. On the other hand, the Moutard-type transforms are successfully used in studies of integrable models in dimension $2+1$ and in the spectral theory in dimension 2 ; see [3], 5], 7], 8]-11] and references therein.

The Moutard-type transforms correspond in quadratures to coefficients and related solutions of an appropriate linear PDE (and its conjugate) on the plane new coefficients and related solutions for this PDE (and its conjugate). In this article we show that this approach is applicable to equation (1). In particular, it allows to study equation (1) in some important cases when $u$ has strong singularities (e.g. contour poles), and, as a corollary, assumptions (2), (3) are not valid at all. It is quite likely that the known methods of the generalized analytic functions theory dot not admit appropriate generalizations for the aforementioned cases.

In addition, our interest to the generalized analytic functions with strong singularities was, in particular, strongly motivated by studies going back to [4].

Note that the present work was stimulated by recent articles [9], 10] by I.A. Taimanov on Moutard-type transforms for two-dimensional Dirac operators with applications to integrable systems in dimension $2+1$ and to differential geometry.

The results of the present work can be summarized as follows:

- We construct Moutard-type transforms for equation (11) considering this equation as a particular case of the two-dimensional Dirac system

$$
\partial_{\bar{z}} \psi_{1}=u \psi_{2}, \quad \partial_{z} \psi_{2}=v \psi_{1} \text { in } D \subseteq \mathbb{C}
$$


where $D$ is the same that in (11). Related algebraic and analytic results in this connection are presented in Section 2 .

- Explicit examples of generalized analytic functions obtained by the Moutard-type transforms from usual holomorphic functions are given in Section 3. These examples include generalized analytic functions with contour poles.

\section{Moutard transform}

We consider the two-dimensional Dirac system (5) and the conjugate system

$$
\partial_{\bar{z}} \psi_{1}^{+}=-v \psi_{2}^{+}, \quad \partial_{z} \psi_{2}^{+}=-u \psi_{1}^{+} \text {in } D \subseteq \mathbb{C} .
$$

If

$$
v=\bar{u},
$$

then system (15) is reduces to (1). More precisely, in this case:

if $\psi$ satisfies (1), then $\left[\begin{array}{l}\psi_{1} \\ \psi_{2}\end{array}\right]=\left[\begin{array}{l}\psi \\ \bar{\psi}\end{array}\right]$ satisfies (5);

if $\left[\begin{array}{l}\psi_{1} \\ \psi_{2}\end{array}\right]$ satisfies (5), then $\psi=\frac{1}{2}\left(\psi_{1}+\bar{\psi}_{2}\right)$ and $\psi=\frac{1}{2 i}\left(\psi_{1}-\bar{\psi}_{2}\right)$ satisfy (11).

In addition, if (7) holds, then the conjugate system (6) is reduced to the equation

$$
\partial_{\bar{z}} \psi^{+}=-\bar{u} \bar{\psi}^{+} \text {in } D \subseteq \mathbb{C} .
$$

In the theory of generalized analytic functions equation (9) is known as the conjugate equation to the initial equation (1); see [12].

Let

$$
\vec{\psi}(j)=\left[\begin{array}{l}
\psi_{1}(z, j) \\
\psi_{2}(z, j)
\end{array}\right], \quad \vec{\psi}^{+}(j)=\left[\begin{array}{c}
\psi_{1}^{+}(z, j) \\
\psi_{2}^{+}(z, j)
\end{array}\right], \quad j=1, \ldots, N,
$$

denote a set of fixed solutions of systems (5) and (6), respectively.

In addition, for systems (5) and (6) , we consider the potentials $\omega_{j, k}=$ $\omega_{j, k}(z)$ defined as follows:

$$
\partial_{z} \omega_{j, k}=\psi_{1}(j) \psi_{1}^{+}(k), \quad \partial_{\bar{z}} \omega_{j, k}=-\psi_{2}(j) \psi_{2}^{+}(k) \text { in } D \subseteq \mathbb{C}, \quad j, k=1, \ldots, N,
$$


under the assumption that $D$ is simply connected. Note that

$\partial_{\bar{z}} \partial_{z} \omega_{j, k}=\left(\partial_{\bar{z}} \psi_{1}(j)\right) \psi_{1}^{+}(k)+\psi_{1}(j)\left(\partial_{\bar{z}} \psi_{1}^{+}(k)\right)=u \psi_{2}(j) \psi_{1}^{+}(k)-v \psi_{1}(j) \psi_{2}^{+}(k)$,

$\partial_{z} \partial_{\bar{z}} \omega_{j, k}=-\left(\partial_{z} \psi_{2}(j)\right) \psi_{2}^{+}(k)-\psi_{2}(j)\left(\partial_{z} \psi_{2}^{+}(k)\right)=-v \psi_{1}(j) \psi_{2}^{+}(k)+u \psi_{2}(j) \psi_{1}^{+}(k)$,

and, thus,

$$
\partial_{\bar{z}} \partial_{z} \omega_{j, k}=\partial_{z} \partial_{\bar{z}} \omega_{j, k}
$$

Therefore, definitions (11) are self-consistent, and the integration constants may depend on the concrete situation.

Let us introduce $N \times N$ matrix $\Omega=\left(\Omega_{k, j}(z)\right)=\left(\omega_{j, k}(z)\right)$ :

$$
\Omega=\left[\begin{array}{cccc}
\omega_{1,1} & \omega_{2,1} & \ldots & \omega_{N, 1} \\
\omega_{1,2} & \omega_{2,2} & \ldots & \omega_{N, 2} \\
\vdots & \vdots & \ddots & \vdots \\
\omega_{1, N} & \omega_{2, N} & \ldots & \omega_{N, N}
\end{array}\right]
$$

Theorem 1 Suppose that $\vec{\psi}(j), \vec{\psi}^{+}(j), j=1, \ldots, N$, (see (10)) are formal solutions of systems (5), (6), respectively, for given coefficients $u=u(z)$, $v=v(z)$, and that $\operatorname{det} \Omega \neq 0$, where $\Omega$ is defined by (11)-(13). Let the transform

$$
\begin{aligned}
& \{u, v\} \rightarrow\{\tilde{u}, \tilde{v}\} \\
& \left\{\vec{\psi}(0), \vec{\psi}^{+}(0)\right\} \rightarrow\left\{\overrightarrow{\tilde{\psi}}(0), \overrightarrow{\tilde{\psi}}^{+}(0)\right\}
\end{aligned}
$$

be defined as follows:

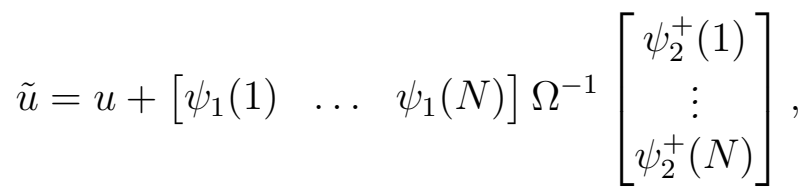

$$
\begin{aligned}
& \tilde{v}=v-\left[\begin{array}{lll}
\psi_{2}(1) & \ldots & \psi_{2}(N)
\end{array}\right] \Omega^{-1}\left[\begin{array}{c}
\psi_{1}^{+}(1) \\
\vdots \\
\psi_{1}^{+}(N)
\end{array}\right] \text {, } \\
& {\left[\begin{array}{c}
\tilde{\psi}_{1}(0) \\
\tilde{\psi}_{2}(0)
\end{array}\right]=\left[\begin{array}{c}
\psi_{1}(0) \\
\psi_{2}(0)
\end{array}\right]-\left[\begin{array}{lll}
\psi_{1}(1) & \ldots & \psi_{1}(N) \\
\psi_{2}(1) & \ldots & \psi_{2}(N)
\end{array}\right] \Omega^{-1}\left[\begin{array}{c}
\omega_{0,1} \\
\vdots \\
\omega_{0, N}
\end{array}\right],}
\end{aligned}
$$




$$
\left[\begin{array}{c}
\tilde{\psi}_{1}^{+}(0) \\
\tilde{\psi}_{2}^{+}(0)
\end{array}\right]=\left[\begin{array}{c}
\psi_{1}^{+}(0) \\
\psi_{2}^{+}(0)
\end{array}\right]-\left[\begin{array}{lll}
\psi_{1}^{+}(1) & \ldots & \psi_{1}^{+}(N) \\
\psi_{2}^{+}(1) & \ldots & \psi_{2}^{+}(N)
\end{array}\right]\left(\Omega^{-1}\right)^{t}\left[\begin{array}{c}
\omega_{1,0} \\
\vdots \\
\omega_{N, 0}
\end{array}\right],
$$

where $\vec{\psi}(0), \vec{\psi}^{+}(0)$ are some formal solutions to systems (5), (6), respectively, $\omega_{0, j}$ and $\omega_{j, 0}$ are defined as in (11), but in terms of $\vec{\psi}(0), \vec{\psi}^{+}(j)$ and $\vec{\psi}(j)$, $\vec{\psi}^{+}(0)$, respectively, and the symbol $t$ in (19) stands for the matrix transposition. Then the transformed functions $\overrightarrow{\tilde{\psi}}(0), \overrightarrow{\tilde{\psi}}^{+}(0)$ solve the transformed Dirac equations:

$$
\begin{array}{lll}
\partial_{\bar{z}} \tilde{\psi}_{1}(0)=\tilde{u} \tilde{\psi}_{2}(0), & \partial_{z} \tilde{\psi}_{2}(0)=\tilde{v} \tilde{\psi}_{1}(0) & \text { in } D, \\
\partial_{\bar{z}} \tilde{\psi}_{1}^{+}(0)=-\tilde{v} \tilde{\psi}_{2}^{+}(0), & \partial_{z} \tilde{\psi}_{2}^{+}(0)=-\tilde{u} \tilde{\psi}_{1}^{+}(0) & \text { in } D .
\end{array}
$$

Historically, transformations like (14)-(15) go back to the paper [6], and are known in the literature as Moutard transforms or Moutard-type transforms. For the two-dimensional Dirac operators the Moutard transforms were considered many times in the literature, see, for example, [5], [9], [10] and references therein. Nevertheless, for completeness of exposition, we present the proof of Theorem 1 in Section 4 .

The point is that the Moutard-type transform (14), (15) admits the following reduction to the case of equations (11), (9), i.e. to the case of generalized analytic functions.

Let

$$
\psi(j)=\psi(z, j), \quad \psi^{+}(j)=\psi^{+}(z, j), \quad j=1, \ldots, N,
$$

denote a set of fixed solutions of (11) and (9), respectively. Then we can define $\omega_{j, k}=\omega_{j, k}(z)$ as imaginary-valued functions satisfying

$$
\partial_{z} \omega_{j, k}=\psi(j) \psi^{+}(k), \quad \partial_{\bar{z}} \omega_{j, k}=-\overline{\psi(j) \psi^{+}(k)} \text { in } D, \quad j, k=1, \ldots, N,
$$

where (23) is the reduction of (11) corresponding to

$$
\psi_{1}(j)=\psi(j), \quad \psi_{2}(j)=\overline{\psi(j)}, \quad \psi_{1}^{+}(j)=\psi^{+}(j), \quad \psi_{2}^{+}(j)=\overline{\psi^{+}(j)} .
$$

Remark 1 It is interesting to note that real-valued function $\phi=\frac{\omega_{j, k}}{2 i}, i=$ $\sqrt{-1}$, for some fixed $j, k$, is known in the generalized analytic functions theory as a potential for equation (11); see [12]. 
Theorem 2 Suppose that $\psi(j), \psi^{+}(j), j=1, \ldots, N$, (see (22)) are formal solutions of equations (11), (9), respectively, for a given coefficient $u=u(z)$, and that $\operatorname{det} \Omega \neq 0$, where $\Omega$ is defined according to (13), (23) with imaginaryvalued $\omega_{j, k}$. Let the transform

$$
\begin{aligned}
& u \rightarrow \tilde{u}, \\
& \left\{\psi(0), \psi^{+}(0)\right\} \rightarrow\left\{\tilde{\psi}(0), \tilde{\psi}^{+}(0)\right\}
\end{aligned}
$$

be defined as follows:

$$
\begin{gathered}
\tilde{u}=u+\left[\begin{array}{lll}
\psi(1) & \ldots & \psi(N)
\end{array}\right] \Omega^{-1}\left[\begin{array}{c}
\overline{\psi^{+}(1)} \\
\vdots \\
\overline{\psi^{+}(N)}
\end{array}\right], \\
\tilde{\psi}(0)=\psi(0)-\left[\begin{array}{lll}
\psi(1) & \ldots & \psi(N)
\end{array}\right] \Omega^{-1}\left[\begin{array}{c}
\omega_{0,1} \\
\vdots \\
\omega_{0, N}
\end{array}\right], \\
\tilde{\psi}^{+}(0)=\psi^{+}(0)-\left[\begin{array}{lll}
\psi^{+}(1) & \ldots & \psi^{+}(N)
\end{array}\right]\left(\Omega^{-1}\right)^{t}\left[\begin{array}{c}
\omega_{1,0} \\
\vdots \\
\omega_{N, 0}
\end{array}\right],
\end{gathered}
$$

where $\psi(0), \psi^{+}(0)$ are some formal solutions to equations (11), (9), respectively, $\omega_{0, j}$ and $\omega_{j, 0}$ are imaginary-valued and are defined as in (23), but in terms of $\psi(0), \psi^{+}(j)$ and $\psi(j), \psi^{+}(0)$, respectively, and $t$ in (29) stands for the matrix transposition. Then the transformed functions $\tilde{\psi}(0), \tilde{\psi}^{+}(0)$ solve the transformed generalized-analytic function equations:

$$
\begin{array}{ll}
\partial_{\bar{z}} \tilde{\psi}(0)=\tilde{u} \overline{\psi(0)} & \text { in } D, \\
\partial_{\bar{z}} \tilde{\psi}^{+}(0)=-\overline{\tilde{u}} \tilde{\psi}^{+}(0) & \text { in } D .
\end{array}
$$

To our best knowledge, Moutard-type type transforms like (25), (26) were not yet considered in the generalized-analytic function theory.

Theorem 2 follows from Theorem 1 in the framework of reductions (7), (24). In this case using also that $\bar{\Omega}=-\Omega$ one can see that the transform (14), (15) preserves symmetries (7), (24).

The algebraic result of Theorem 2 admits, in particular, the following analytic realization: 
Theorem 3 Let $D$ be an open simply-connected bounded domain in $\mathbb{C}$ with $C^{1}$-boundary and $u$ satisfy (2). Let $\psi(j) \in W^{1, p}(D)$ and $\psi^{+}(j) \in W^{1, p}(D)$, $j=1, \ldots, N$, be solutions of equations (11) and (9), respectively, and $\operatorname{det} \Omega \neq$

0 in $D \cup \partial D$, where $\Omega$ is defined according to (13), (23) with imaginary-valued $\omega_{j, k}$. Let the Moutard transform be defined according the formulas (25)-(29) as in Theorem Q2.

Then the transformed coefficient $\tilde{u}$ satisfied (2) as well as the initial u.

In Theorem 3, $W^{1, p}$ denotes the standard Sobolev space.

Theorem 3 follows from formula (27) and from the properties that $\psi(j)$, $\psi^{+}(j), j=1, \ldots, N$, and $\omega_{j, k}, 1 \leq j, k \leq N$, and $\operatorname{det} \Omega$ are continuous in $D \cup \partial D$. Note that, in order to obtain the aforementioned continuity of $\psi(j), \psi^{+}(j)$, we use that, under our assumptions on $D$, if $f \in W^{1, p}(D)$ then $f \in C^{\alpha}(D \cup \partial D)$, where $C^{\alpha}$ denotes the standard Hölder space, $\alpha=(p-2) / p$ (see [1]). Finally, the continuity of $\omega_{j, k}$ follows from the continuity of $\psi(j)$, $\psi^{+}(k)$ and from formula (11).

\section{$3 \quad$ Explicit examples}

In this section we present explicit examples illustrating the Moutard-type transforms for generalized analytic functions, i.e. transforms (25) $-(\underline{29})$.

Let

$$
\begin{aligned}
& u \equiv 0, \quad N=1, \quad \psi(1)=f(z), \quad \psi^{+}(1)=f^{+}(z), \\
& \psi(0)=\psi(z, 0), \quad \psi^{+}(0)=\psi^{+}(z, 0),
\end{aligned}
$$

where $f, f^{+}$and $\psi(0), \psi^{+}(0)$ are holomorphic on $D$. Then:

1. For imaginary-valued $\omega_{1,1}$ and $\omega_{0,1}, \omega_{1,0}$ of (23) and (28), (29), we have

$$
\begin{aligned}
& \omega_{1,1}(z)=F_{1,1}(z)-\overline{F_{1,1}(z)}+c_{1,1}, \\
& \omega_{0,1}(z)=F_{0,1}(z)-\overline{F_{0,1}(z)}+c_{0,1}, \quad \omega_{1,0}(z)=F_{1,0}(z)-\overline{F_{1,0}(z)}+c_{1,0}
\end{aligned}
$$

where $F_{1,1}$ and $F_{0,1}, F_{1,0}$ are holomorphic functions on $D$ such that

$$
\begin{aligned}
& \partial_{z} F_{1,1}(z)=f(z) f^{+}(z), \\
& \partial_{z} F_{0,1}(z)=\psi(z, 0) f^{+}(z), \quad \partial_{z} F_{1,0}(z)=f(z) \psi^{+}(z, 0),
\end{aligned}
$$

and $c_{1,1}, c_{0,1}, c_{1,0}$ are pure imaginary constants; 
2. Formulas (27)-(29) take the form:

$$
\begin{gathered}
\tilde{u}(z)=\frac{f(z) \overline{f^{+}(z)}}{\omega_{1,1}(z)} \\
\tilde{\psi}(z, 0)=\psi(z, 0)-f(z) \frac{\omega_{0,1}(z)}{\omega_{1,1}(z)}, \\
\tilde{\psi}^{+}(z, 0)=\psi^{+}(z, 0)-f^{+}(z) \frac{\omega_{1,0}(z)}{\omega_{1,1}(z)} .
\end{gathered}
$$

In addition, equations (30), (31) for $\tilde{\psi}(0), \tilde{\psi}^{+}(0)$ are nontrivial already for

$$
f(z)=C \neq 0, \quad f^{+}(z)=C^{+} \neq 0
$$

where $C, C^{+}$are complex constants. In particular, in this case

$$
\tilde{u}(z)=\frac{C \overline{C^{+}}}{2 i \operatorname{Im}\left(C C^{+} z\right)+c_{1,1}} .
$$

One can see that this $\tilde{u}$ satisfies (2) if $(D \cup \partial D) \subset \Lambda^{+}$or $(D \cup \partial D) \subset \Lambda^{-}$, where

$$
\begin{aligned}
& \Lambda^{+}=\left\{z \in \mathbb{C}: \operatorname{Im}\left(2 C C^{+} z+c_{1,1}\right)>0\right\} \\
& \Lambda^{-}=\left\{z \in \mathbb{C}: \operatorname{Im}\left(2 C C^{+} z+c_{1,1}\right)<0\right\}
\end{aligned}
$$

However, this $\tilde{u}$ does not satisfy (2) at all if $D \cap \mathcal{L} \neq \emptyset$, where

$$
\mathcal{L}=\left\{z \in \mathbb{C}: \operatorname{Im}\left(2 C C^{+} z+c_{1,1}\right)=0\right\}
$$

The point is that already the Moutard transform (25)-(29) for the case (32), (38) yields generalized analytic functions with contour poles: with poles on $\mathcal{L}$ in this particular case.

Next, we consider the example when

$$
\begin{aligned}
& u \equiv 0, \quad N=2, \quad \psi(1)=f_{1}, \quad \psi^{+}(1)=f_{1}^{+}, \quad \psi(2)=f_{2}, \quad \psi^{+}(2)=f_{2}^{+}, \\
& \psi(0)=\psi(z, 0), \quad \psi^{+}(0)=\psi^{+}(z, 0),
\end{aligned}
$$

where $f_{1}, f_{1}^{+}, f_{2}, f_{2}^{+}$are complex constants, and $\psi(0), \psi^{+}(0)$ are holomorphic functions on $D$. In this case: 
1. For imaginary-valued $\omega_{j, k}$ of (23), (28), (29), $j, k \in\{0,1,2\}$, we have

$$
\begin{aligned}
& \omega_{j, k}(z)=f_{j} f_{k}^{+} z-\overline{f_{j} f_{k}^{+} z}+c_{j, k}, \\
& \omega_{0, k}(z)=\Psi(z) f_{k}^{+}-\overline{\Psi(z) f_{k}^{+}}+c_{0, k}, \quad \omega_{j, 0}(z)=f_{j} \Psi^{+}(z)-\overline{f_{j} \Psi^{+}(z)}+c_{j, 0},
\end{aligned}
$$

where $j, k=1,2, \Psi, \Psi^{+}$are holomorphic functions on $D$ such that

$$
\partial_{z} \Psi(z)=\psi(z, 0), \quad \partial_{z} \Psi^{+}(z)=\psi^{+}(z, 0),
$$

and $c_{j, k}$ are pure imaginary constants, $j, k=0,1,2$;

2. Formulas (27)-(29) take the form:

$$
\begin{gathered}
\tilde{u}(z)=\frac{f_{1} \overline{f_{1}^{+}} c_{2,2}-f_{2} \overline{f_{1}^{+}} c_{1,2}-f_{1} \overline{f_{2}^{+}} c_{2,1}+f_{2} \overline{f_{2}^{+}} c_{1,1}}{\operatorname{det} \Omega}, \\
\tilde{\psi}(z, 0)=\psi(z, 0)-(\operatorname{det} \Omega)^{-1} \times \\
\times\left(\left[\left(-f_{1} \overline{f_{2} f_{2}^{+}}+f_{2} \overline{f_{1} f_{2}^{+}}\right) \bar{z}+f_{1} c_{2,2}-f_{2} c_{1,2}\right] \omega_{0,1}(z)+\right. \\
\left.+\left[\left(f_{1} \overline{f_{2} f_{1}^{+}}-f_{2} \overline{f_{1} f_{1}^{+}}\right) \bar{z}-f_{1} c_{2,1}+f_{2} c_{1,1}\right] \omega_{0,2}(z)\right), \\
\tilde{\psi}^{+}(z, 0)=\psi^{+}(z, 0)-(\operatorname{det} \Omega)^{-1} \times \\
\times\left(\left[\left(-f_{1}^{+} \overline{f_{2} f_{2}^{+}}+f_{2}^{+} \overline{f_{2} f_{1}^{+}}\right) \bar{z}+f_{1}^{+} c_{2,2}-f_{2}^{+} c_{2,1}\right] \omega_{1,0}(z)+\right. \\
\left.+\left[\left(f_{1}^{+} \overline{f_{1} f_{2}^{+}}-f_{2}^{+} \overline{f_{1} f_{1}^{+}}\right) \bar{z}-f_{1}^{+} c_{1,2}+f_{2}^{+} c_{1,1}\right] \omega_{2,0}(z)\right),
\end{gathered}
$$

where

$$
\begin{aligned}
& \operatorname{det} \Omega=2 \operatorname{Re}\left[\left(f_{2} f_{1}^{+} \overline{f_{1} f_{2}^{+}}-f_{1} f_{1}^{+} \overline{f_{2} f_{2}^{+}}\right) z \bar{z}+\right. \\
& \left.+\left(c_{2,2} f_{1} f_{1}^{+}+c_{1,1} f_{2} f_{2}^{+}-c_{1,2} f_{2} f_{1}^{+}-c_{2,1} f_{1} f_{2}^{+}\right) z\right]+c_{1,1} c_{2,2}-c_{2,1} c_{1,2}
\end{aligned}
$$

$\Omega$ is the matrix defined according to (13) for $N=2$.

Note that if

$$
\begin{aligned}
& c_{2,2} f_{1} f_{1}^{+}+c_{1,1} f_{2} f_{2}^{+}-c_{1,2} f_{2} f_{1}^{+}-c_{2,1} f_{1} f_{2}^{+}=0, \\
& f_{1} \overline{f_{1}^{+}} c_{2,2}-f_{2} \overline{f_{1}^{+}} c_{1,2}-f_{1} \overline{f_{2}^{+}} c_{2,1}+f_{2} \overline{f_{2}^{+}} c_{1,1} \neq 0, \\
& \operatorname{Re}\left(f_{2} f_{1}^{+} \overline{f_{1} f_{2}^{+}}-f_{1} f_{1}^{+} \overline{f_{2} f_{2}^{+}}\right) \neq 0,
\end{aligned}
$$


then $\tilde{u}$ in (45) is spherically-symmetric and non-trivial. In addition, assuming that

$$
\sigma \geq 0, \text { where } \sigma=-\frac{c_{1,1} c_{2,2}-c_{2,1} c_{1,2}}{2 \operatorname{Re}\left(f_{2} f_{1}^{+} \overline{f_{1} f_{2}^{+}}-f_{1} f_{1}^{+} \overline{f_{2} f_{2}^{+}}\right)},
$$

we have that: $\tilde{u}$ has a pole on

$$
\begin{gathered}
S_{r}=\left\{z \in \mathbb{C}:|z|^{2}=r\right\}, \text { where } r^{2}=\sigma ; \\
\tilde{u}(z)=O\left(|z|^{-2}\right) \text { as }|z| \rightarrow \infty .
\end{gathered}
$$

For example, assumptions (49) are fulfilled if

$f_{1}=1, f_{1}^{+}=1, \quad f_{2}=i, f_{2}^{+}=i, \quad c_{1,1}=c_{2,2}, \quad c_{1,2}=-c_{2,1}, \quad\left|c_{1,1}\right|^{2}+\left|c_{1,2}\right|^{2} \neq 0$.

One can see that the Moutard transform (25)-(29) for the case (42), (49), (50) yields again generalized analytic functions with contour poles: with poles on $S_{r}$ in this particular case.

Actually, our Moutard-type transforms (25)-(29) give a method for developing a proper theory of generalized analytic functions with contour and point poles of some natural class. This issue will be developed in the subsequent work.

\section{Proof of Theorem 1}

Using (11),(13) we obtain:

$$
\begin{aligned}
& \partial_{z} \Omega=\left[\begin{array}{c}
\psi_{1}^{+}(1) \\
\vdots \\
\psi_{1}^{+}(N)
\end{array}\right]\left[\begin{array}{lll}
\psi_{1}(1) & \ldots & \left.\psi_{1}(N)\right]
\end{array}\right.
\end{aligned}
$$

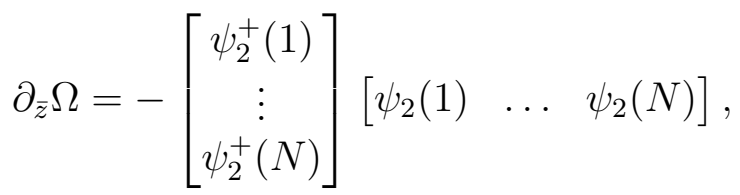

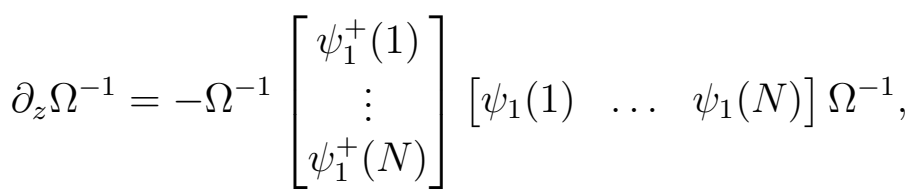




$$
\partial_{\bar{z}} \Omega^{-1}=\Omega^{-1}\left[\begin{array}{c}
\psi_{2}^{+}(1) \\
\vdots \\
\psi_{2}^{+}(N)
\end{array}\right]\left[\begin{array}{lll}
\psi_{2}(1) & \ldots & \left.\psi_{2}(N)\right] \Omega^{-1} .
\end{array}\right.
$$

Using (18) and (5), (11), (56), (57), we obtain:

$$
\begin{aligned}
& \partial_{\bar{z}} \tilde{\psi}_{1}(0)=\partial_{\bar{z}} \psi_{1}(0)-\left[\begin{array}{lll}
\partial_{\bar{z}} \psi_{1}(1) & \ldots & \partial_{\bar{z}} \psi_{1}(N)
\end{array}\right] \Omega^{-1}\left[\begin{array}{c}
\omega_{0,1} \\
\vdots \\
\omega_{0, N}
\end{array}\right]+
\end{aligned}
$$

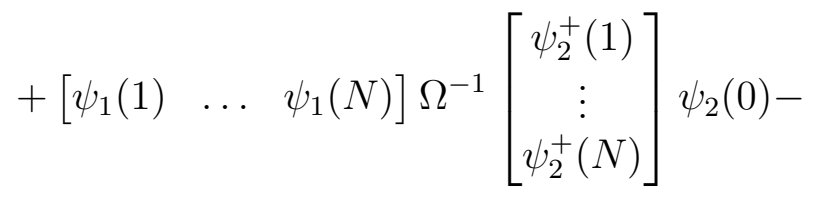

$$
\begin{aligned}
& -\left[\begin{array}{lll}
\psi_{1}(1) & \ldots & \psi_{1}(N)
\end{array}\right] \Omega^{-1}\left[\begin{array}{c}
\psi_{2}^{+}(1) \\
\vdots \\
\psi_{2}^{+}(N)
\end{array}\right]\left[\begin{array}{lll}
\psi_{2}(1) & \ldots & \psi_{2}(N)
\end{array}\right] \Omega^{-1}\left[\begin{array}{c}
\omega_{0,1} \\
\vdots \\
\omega_{0, N}
\end{array}\right]= \\
& =\left(u+\left[\begin{array}{lll}
\psi_{1}(1) & \ldots & \left.\psi_{1}(N)\right]
\end{array}\right] \Omega^{-1}\left[\begin{array}{c}
\psi_{2}^{+}(1) \\
\vdots \\
\psi_{2}^{+}(N)
\end{array}\right]\right) \times
\end{aligned}
$$

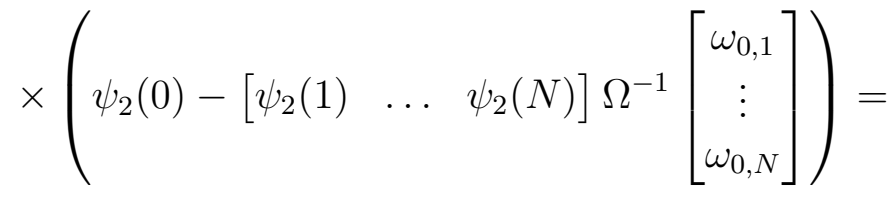

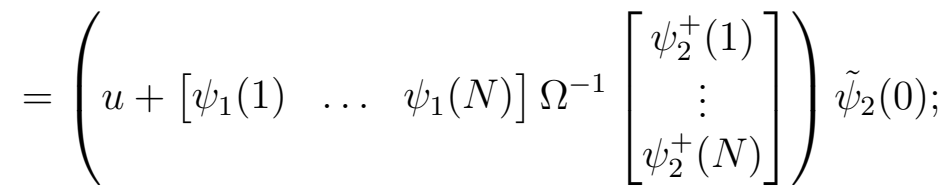

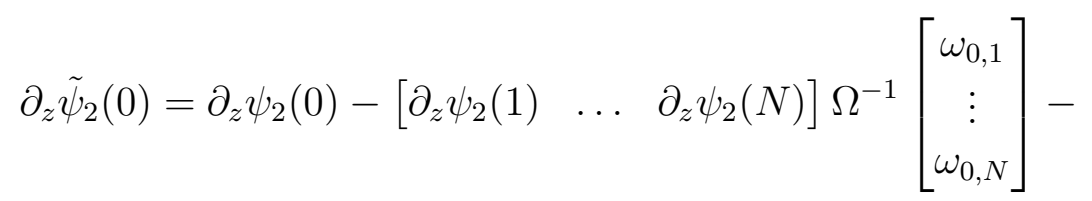

$$
\begin{aligned}
& -\left[\begin{array}{lll}
\psi_{2}(1) & \ldots & \psi_{2}(N)
\end{array}\right] \Omega^{-1}\left[\begin{array}{c}
\psi_{1}^{+}(1) \\
\vdots \\
\psi_{1}^{+}(N)
\end{array}\right] \psi_{1}(0)+
\end{aligned}
$$




$$
\begin{aligned}
& +\left[\begin{array}{lll}
\psi_{2}(1) & \ldots & \psi_{2}(N)
\end{array}\right] \Omega^{-1}\left[\begin{array}{c}
\psi_{1}^{+}(1) \\
\vdots \\
\psi_{1}^{+}(N)
\end{array}\right]\left[\begin{array}{lll}
\psi_{1}(1) & \ldots & \psi_{1}(N)
\end{array}\right] \Omega^{-1}\left[\begin{array}{c}
\omega_{0,1} \\
\vdots \\
\omega_{0, N}
\end{array}\right]= \\
& =\left(\begin{array}{lll}
v-\left[\psi_{2}(1)\right. & \ldots & \left.\psi_{2}(N)\right]
\end{array} \Omega^{-1}\left[\begin{array}{c}
\psi_{1}^{+}(1) \\
\vdots \\
\psi_{1}^{+}(N)
\end{array}\right]\right) \times
\end{aligned}
$$

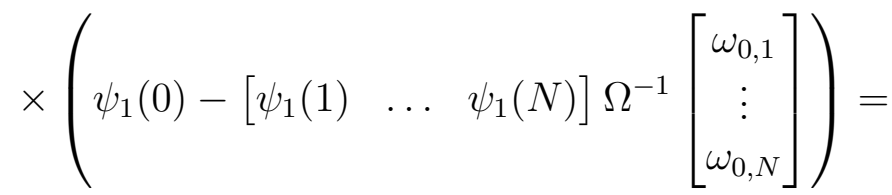

$$
\begin{aligned}
& \left.=\left(\begin{array}{lll}
v-\left[\psi_{2}(1)\right. & \ldots & \psi_{2}(N)
\end{array}\right] \Omega^{-1}\left[\begin{array}{c}
\psi_{1}^{+}(1) \\
\vdots \\
\psi_{1}^{+}(N)
\end{array}\right]\right) \tilde{\psi}_{1}(0) .
\end{aligned}
$$

Formulas (58), (59) and (16), (17) imply (20).

Using (19) and (5), (11), (56)), (57), and the formulas

$$
\partial_{z}\left(\Omega^{-1}\right)^{t}=\left(\partial_{z} \Omega^{-1}\right)^{t}, \quad \partial_{\bar{z}}\left(\Omega^{-1}\right)^{t}=\left(\partial_{\bar{z}} \Omega^{-1}\right)^{t},
$$

we obtain:

$$
\begin{aligned}
& \partial_{\bar{z}} \tilde{\psi}_{1}^{+}(0)=\partial_{\bar{z}} \psi_{1}^{+}(0)-\left[\begin{array}{lll}
\partial_{\bar{z}} \psi_{1}^{+}(1) & \ldots & \partial_{\bar{z}} \psi_{1}^{+}(N)
\end{array}\right]\left(\Omega^{-1}\right)^{t}\left[\begin{array}{c}
\omega_{1,0} \\
\vdots \\
\omega_{N, 0}
\end{array}\right]+ \\
& +\left[\begin{array}{lll}
\psi_{1}^{+}(1) & \ldots & \psi_{1}^{+}(N)
\end{array}\right]\left(\Omega^{-1}\right)^{t}\left[\begin{array}{c}
\psi_{2}(1) \\
\vdots \\
\psi_{2}(N)
\end{array}\right] \psi_{2}^{+}(0)- \\
& -\left[\begin{array}{lll}
\psi_{1}^{+}(1) & \ldots & \psi_{1}^{+}(N)
\end{array}\right]\left(\Omega^{-1}\right)^{t}\left[\begin{array}{c}
\psi_{2}(1) \\
\vdots \\
\psi_{2}(N)
\end{array}\right]\left[\begin{array}{lll}
\psi_{2}^{+}(1) & \ldots & \psi_{2}^{+}(N)
\end{array}\right]\left(\Omega^{-1}\right)^{t}\left[\begin{array}{c}
\omega_{1,0} \\
\vdots \\
\omega_{N, 0}
\end{array}\right]=
\end{aligned}
$$

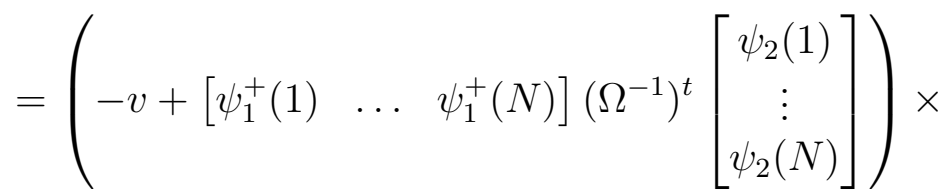




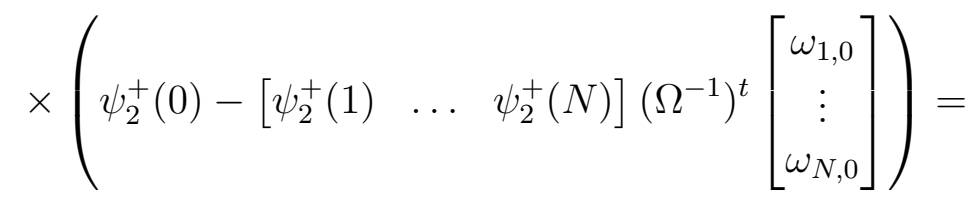

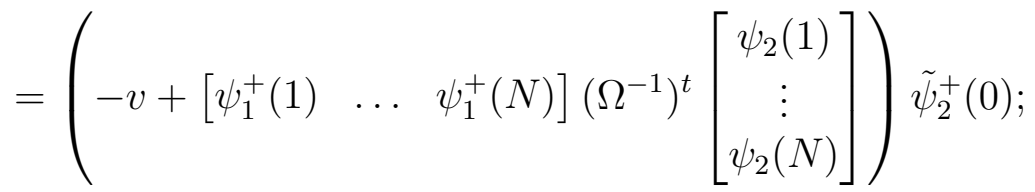

and

$$
\begin{aligned}
& \partial_{z} \tilde{\psi}_{2}^{+}(\lambda)=\partial_{z} \psi_{2}^{+}(\lambda)-\left[\begin{array}{lll}
\partial_{z} \psi_{2}^{+}(1) & \ldots & \partial_{z} \psi_{2}^{+}(N)
\end{array}\right]\left(\Omega^{-1}\right)^{t}\left[\begin{array}{c}
\omega_{1,0} \\
\vdots \\
\omega_{N, 0}
\end{array}\right]- \\
& -\left[\begin{array}{lll}
\psi_{2}^{+}(1) & \ldots & \psi_{2}^{+}(N)
\end{array}\right]\left(\Omega^{-1}\right)^{t}\left[\begin{array}{c}
\psi_{1}(1) \\
\vdots \\
\psi_{1}(N)
\end{array}\right] \psi_{1}^{+}(0)+ \\
& +\left[\begin{array}{lll}
\psi_{2}^{+}(1) & \ldots & \psi_{2}^{+}(N)
\end{array}\right]\left(\Omega^{-1}\right)^{t}\left[\begin{array}{c}
\psi_{1}(1) \\
\vdots \\
\psi_{1}(N)
\end{array}\right]\left[\begin{array}{lll}
\psi_{1}^{+}(1) & \ldots & \psi_{1}^{+}(N)
\end{array}\right]\left(\Omega^{-1}\right)^{t}\left[\begin{array}{c}
\omega_{1,0} \\
\vdots \\
\omega_{N, 0}
\end{array}\right]=
\end{aligned}
$$

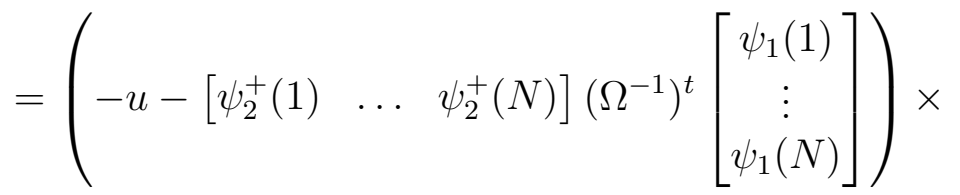

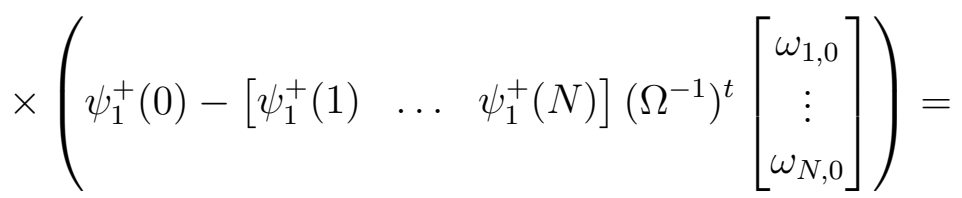

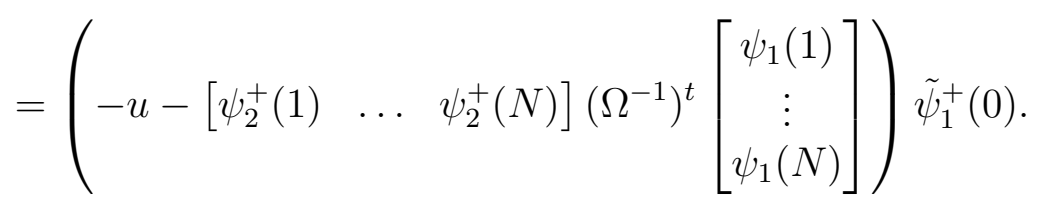

Formulas (60), (61) and (16), (17) imply (21).

This completes the proof of Theorem 1 . 


\section{References}

[1] R. Adams, J. Fournier, Sobolev spaces, Academic Press, 2003.

[2] L. Bers, Theory of pseudo-analytic functions, Courant Institute of Mathematical Sciences, New York University, Institute for Mathematics and Mechanics, 1953, 187 pages.

[3] A. Doliwa, P. Grinevich, M. Nieszporski and P.M. Santini, Integrable lattices and their sublattices: From the discrete Moutard (discrete Cauchy-Riemann) 4-point equation to the self-adjoint 5-point scheme, J. Math. Phys., 48 (2007), p. 013513.

[4] P.G. Grinevich, S.P. Novikov, Two-dimensional 'inverse scattering problem' for negative energies and generalized-analytic functions. 1. Energies below the ground state, Functional Analysis and Its Applications, 22 (1988), pp. 19-27.

[5] V.B. Matveev, M.A. Salle, Darboux transformations and solitons, Springer Series in Nonlinear Dynamics. Springer-Verlag, Berlin, 1991.

[6] T.F. Moutard, Sur la construction des équations de la forme $\frac{1}{z} \frac{\partial^{2} z}{\partial x \partial y}=$ $\lambda(x, y)$ qui admettenent une intégrale générale explicite. J. École Polytechnique, 45 (1878), pp. 1-11.

[7] J.J.C. Nimmo, W.K. Schief, Superposition principles associated with the Moutard transformation: an integrable discretization of a 2+1dimensional sine-Gordon system, Proc. R. Soc. London A, 453 (1997), pp. 255-279.

[8] R.G. Novikov, I.A. Taimanov, S.P. Tsarev, Two-dimensional von Neumann-Wigner potentials with a multiple positive eigenvalue, Functional Analysis and Its Applications, 48:4 (2014), pp. 295-297.

[9] I.A. Taimanov, Blowing up solutions of the modified Novikov-Veselov equation and minimal surfaces, Theoretical and Mathematical Physics, 182:2 (2015), pp. 173-181.

[10] I.A. Taimanov, The Moutard transformation of two-dimensional Dirac operators and Möbius geometry, Mathematical Notes, 97:1 (2015), pp. $124-135$. 
[11] I.A. Taimanov, S.P. Tsarev, On the Moutard transformation and its applications to spectral theory and Soliton equations, Journal of Mathematical Sciences, 170:3 (2010), pp. 371-387.

[12] I.N. Vekua, Generalized Analytic Functions, Pergamon Press Ltd. 1962. 